\title{
La comunicación social de la ciencia a través del etiquetado de alimentos saludables en los EEUU y Europa
}

\author{
Public Communication of Science through healthy food \\ labelling in the US and Europe
}

\author{
Noemí Sanz Merino \\ Universitat de les Illes Balears \\ https://orcid.org/oooo-0002-5093-3883 \\ noemi.sanz@uib.es
}

Resumen: Análisis teórico de las regulaciones de las declaraciones de salud estadounidense y europea. Desde los estudios sobre comunicación y comprensión públicas de la ciencia, identificamos las estrategias base de sus normativas sobre presentación de este tipo de información en el etiquetado alimentario. Atendiendo a investigaciones empíricas sobre percepción e intención de compra, valoramos en qué medida sus enfoques comunicativos ayudan a alcanzar los objetivos reguladores. Resultados: ambas regulaciones tienen diferencias de enfoque dependientes de distintos objetivos políticos; los estudios disponibles apuntan al fracaso de tales objetivos en ambos casos; ello parece deberse a subyacentes concepciones clásicas sobre comunicación y enculturación científicas.

Palabras clave: declaraciones de salud, etiquetado, comunicación científica, compresión pública de la ciencia, intención de compra.

\begin{abstract}
A theoretical analysis of the American and European Health claims regulations. Following the studies on public communication and understanding of science, I identify the underlying regulatory strategies for the regulations on the presentation of this kind of information on food labelling. Based on empirical studies on perception and purchase intention, I assess the extent to which their communicative approaches contribute to achieving the regulatory objectives. Results: Both regulations follow different approaches depending on their different political objectives; The available studies point to the failure of these objectives in both cases; This seems to be due to underlying classical conceptions about science communication and enculturation.
\end{abstract}

Keywords: health claims, labelling, science communication, public understanding of science, purchase intention.

Recibido: 5 de abril de 2020

Aceptado con modificaciones: 28 de julio de 2020

Aceptado: 10 de septiembre de 2020

*Financiación: Este trabajo no habría sido posible sin el apoyo financiero del Fondo Europeo de Desarrollo Regional de la Comisión Europea (FEDER) / Ministerio de Ciencia, Innovación y Universidades de España - Agencia Estatal de Investigación (AEI) / Proyecto de Investigación "Estándares de prueba y elecciones metodológicas en la fundamentación científica de las declaraciones de salud", FFI2017-83543-P, y la ayuda Cas18/o0126. 


\section{Introducción}

"Contribuye al funcionamiento normal del sistema inmunitario", afirmado de una sustancia alimenticia en el etiquetado de un alimento, es una declaración sobre propiedades saludables de los alimentos de uso general (o "declaración de salud""Health claim"). Constituye información nutricional basada en dictámenes científicos, en concreto, afirma una relación entre un alimento específico y una mejora en la salud humana o en la prevención de una enfermedad. Hoy día, este tipo de mensajes están regulados desde las administraciones públicas con la intención, entre otras, de servir de apoyo en la toma de decisiones de los ciudadanos sobre alimentación. La regulación sobre la difusión de las declaraciones de salud constituye un fenómeno susceptible de análisis en términos de comunicación social de la ciencia, como veremos, por varios motivos y, en tanto tal, es el objeto de interés de este trabajo.

Cada vez hay más estudios y revisiones sobre este tipo de regulaciones y su impacto en el consumo. Algunos de los centrados en el desarrollo de esta regulación en general o en cuestiones científicas y epistémicas subyacentes son Lalor y Wall (2011), Biesalski et al. (2011), Boobis et al. (2013), Todt y Luján (2017), González-Díaz, Gil-González y Álvarez-Dardet (2018), Jukola (2019), Luján y Todt (2020). Entre los análisis sobre las actitudes y percepción de los consumidores, se encontrarían, e.g. Nocella y Kennedy (2012), Berhaupt-Glickstein y Hallman (2017) y Hieke y Grunert (2018). El análisis teórico de este trabajo se desarrolla desde el marco de los estudios interesados por la comprensión pública (e.g. Durant et al. 2000; Bauer et al. 2007) y la comunicación social de la ciencia (e.g. Lewenstein, 1995; Bucchi, 2008), situándonos en el ámbito de la producción científica al servicio de la elaboración o implementación de políticas públicas (Jasanoff, 2005; Renn, 2008; López Cerezo, 2018).

El objetivo general es analizar el potencial de este tipo de comunicación de la ciencia para el logro efectivo de los objetivos perseguidos por las regulaciones estadounidense (US-FDA-NLA-1990) y europea (Regulation-EU-1924/2006) sobre el uso de estas declaraciones. ${ }^{1}$ Como veremos, la regulación europea y la norteamericana implementan la comunicación de este tipo de información sobre beneficios desde dos estrategias distintas. Sin embargo, según los estudios empíricos disponibles, ambas regulaciones fracasan en el logro de sus objetivos. Finalmente, concluiremos que ello pudiera deberse a que, pese a sus importantes diferencias, ambas regulaciones parecen igualmente responder a supuestos clásicos sobre los procesos de comunicación y enculturación científicas.

\footnotetext{
${ }^{1}$ Las regulaciones de EE.UU. y la UE, al menos y actualmente, distinguen como subtipos de declaraciones de propiedades saludables (Health claims) las relativas al mantenimiento de la salud (junto con la mejora de la salud) y las de reducción del riesgo de enfermedades (e.g., "Reduce el colesterol"). Se tratan de diferencias en torno al tipo de funcionalidad expresada. En Europa, e.g., también se distinguen a este respecto las declaraciones de salud relacionadas con el desarrollo y crecimiento en niños. Por tales funcionalidades relacionadas con la salud es que se diferencian, e.g., de las "declaraciones nutricionales" u otras alimentarias posibles. En cualquier caso, como veremos, las subcategorías de declaraciones de salud que serán relevantes para nosotros se basan en los criterios evidenciarios a los que está sujeta su respectiva autorización.
} 


\section{Objetivos}

La principal aportación del presente trabajo al estudio de las regulaciones sobre el uso de declaraciones de salud reside en que analiza teóricamente el potencial de las normativas según el enfoque de los Estudios sociales de la ciencia (o "Science \& Technology Studies", STS), en concreto, en materia de comunicación y compresión sociales de la ciencia (estudios sobre Public Understanding of Science, PUS) (véase aparado 4).

La investigación parte de la hipótesis de que el potencial de las regulaciones europea y norteamericana, para el logro de sus respectivos objetivos sociales, es de partida limitado por basar sus estrategias reguladoras en supuestos erróneos sobre comunicación y percepción sociales de la ciencia. Para corroborar esta tesis, se establecieron los siguientes objetivos específicos:

- Establecer la posible correlación de los objetivos políticos específicos perseguidos por cada una de las regulaciones con sus respectivas estrategias reguladoras.

- Identificar el/los modelos de comunicación seguidos en el establecimiento de las directrices impuestas sobre el etiquetado con declaraciones ("Health claims") como parte de dichas estrategias.

- Valorar la adecuación de los enfoques comunicativos seguidos para el logro de aquellos objetivos a la luz del impacto real del uso de las declaraciones de salud en los hábitos de consumo, así como de otros hallazgos relevantes al caso aportados por estudios especializados en comunicación social de la ciencia.

Para alcanzar estos objetivos, se ha realizado una investigación documental sobre las normativas norteamericana y europea. Además, por un lado, se han tenido en cuenta trabajos especializados recientes por analizar en detalle y/o comparar ambas legislaciones según ciertos aspectos, especialmente epistėmicos, que se han desvelado como particularmente relevantes. Al respecto, únicamente se han considerado los que, al menos, versan sobre las evaluación y regulación de declaraciones bajo la consideración de "Health claims". Por otro, consideramos los resultados de investigaciones empíricas sobre compresión, percepción e intención de compra en torno a las mismas declaraciones de salud.

Estos tipos de trabajos especializados han sido obtenidos mediante búsqueda en las bases de datos de publicaciones PubMed, ScienceDirect, Web of Science y Google Scholar, así como por el subsiguiente seguimiento de citas. Respecto de los análisis o revisiones sobre las regulaciones, se han descartado los referidos a declaraciones sobre sustancias alimenticias concretas o dirigidas a grupos poblacionales específicos. Sin embargo, respecto de los estudios del impacto en los consumidores, dada la todavía limitada disponibilidad y variedad de los mismos, no se han hecho tales disquisiciones. 


\section{Las regulaciones estadounidense y europea sobre declaraciones de salud}

Antes de estar regulados, los productos alimentarios que supuestamente tenían propiedades saludables especiales ya se etiquetaban y anunciaban con tal funcionalidad. Algunas administraciones consideraron que el hecho de que ello pudiera responder a estrategias de marketing sin fundamento científico podía conducir a prácticas de mercado injustas y a la desconfianza de los consumidores en las autoridades competentes y la propia ciencia.

En 1990, se proponía la Ley de etiquetado y educación nutricional estadounidense. Más tarde, la Unión Europea siguió los términos establecidos por esta primera normativa con su adopción, en 2006, del Reglamento sobre declaraciones nutricionales y de propiedades saludables en los alimentos. Ambas adopciones regulatorias establecieron que el uso de declaraciones de salud debía basarse en evidencia científica suficiente, i.e. la declaración ha de estar científicamente substanciada. En la Unión Europea (UE), es la Autoridad Europea de Seguridad Alimentaria (EFSA, por sus siglas en inglés) la entidad a cargo de evaluar si una declaración de salud ("Health claim”) está suficientemente justificada. Mientras que, en los Estados Unidos (EEUU), lo es la Administración Federal de Alimentos y Medicamentos (FDA).

La FDA y la EFSA, para juzgar positivamente una solicitud, también deben de valorar si la declaración y las condiciones de consumo que optimizan el beneficio podrán ser entendidas inequívocamente por los consumidores. Su compresión se destaca en ambas regulaciones como un requisito fundamental, ya que el objetivo último compartido es alcanzar que la sociedad tenga hábitos alimentarios más saludables, partiéndose del hecho de que los ciudadanos han de estar bien informados sobre qué productos son especialmente beneficiosos para su salud.

\subsection{Evaluación de beneficios de los alimentos}

Con respecto a la tarea de evaluar la substanciación de los beneficios afirmados en una declaración de salud, los reguladores impusieron que las agencias evaluadoras siguieran un "acuerdo científico significativo" (US-FDA-NLA-1990) o "el estándar más alto posible” (Regulation-EU-1924/2006). Al seguir tal instrucción, la FDA y la EFSA consideraron que ello quería decir que lo que los solicitantes tenían que justificar era la relación causa-efecto entre el consumo del alimento y el resultado sobre la salud declarado. Se estableció que el tipo de pruebas científicas que debían aportarse tenían que basarse en ensayos de control aleatorio (Radomized control trials-RCT) con humanos.

En la Unión Europea se sigue aún este dictamen de causalidad como prerrequisito para lograr una evaluación positiva (ESFA-NDA et al., 2017), aunque ello ya ha generado cierta controversia entre los reguladores europeos y algunos científicos de la nutrición (e.g., Biesalski et al., 2011; Gregori y Gafare, 2012). Estos critican este tipo de imposición normativa, en tanto estándar científico más alto, afirmando que los RCT no es la metodología óptima para la obtención de datos en ciencias de la nutrición. También se argumenta que este enfoque evaluador se transfirió acríticamente desde el campo de la evaluación de beneficios de los medicamentos. En 
cambio y según ellos, a diferencia de los farmacológicos, los beneficios alimenticios son poco pronunciados y se dan a largo plazo, de ahí que otras metodologías científicas (como las epidemiológicas o mecanísticas) no solo sean más recomendables, sino que son las más habituales del campo. ${ }^{2}$

Con tal restringido enfoque evaluador, se estaría siguiendo la estrategia inversa a la típica de la evaluación de riesgos. Mientras, tradicionalmente, esta parte del supuesto de que la permisividad con falsos negativos es perjudicial en la transferencia social de productos científico-tecnológicos, en esta evaluación de beneficios se parte de la consideración de que lo más beneficioso para la sociedad es que no lleguen al mercado alimentos que dicen ser especialmente beneficiosos sin serlo (Luján y Todt, 2020). Es decir, se busca la no autorización de ningún posible falso positivo, lo que se cree que permitirá asegurar la confianza social en las fuentes oficiales y científicas, así como respaldar la innovación alimentaria (Regulation-EU-1924/2006).

Sin embargo, la regulación de EEUU al respecto ha evolucionado considerablemente hasta el punto de haber cambiado su enfoque evaluador. En la actualidad, esta responde a varias reformas introducidas con los años. La más destacada con respecto a nuestro interés fue la causada por la Iniciativa de información sobre salud para una mejor nutrición del consumidor3 (cuyas directrices están actualmente en vigor). Desde entonces, aunque los RCT son aún considerados la prueba evidenciaría de más alto nivel, el estándar evaluador norteamericano sigue un pluralismo metodológico llamado "peso de la evidencia" ("weight of evidence"). Es decir, la FDA acepta también otras metodologías científicas para la substanciación de las declaraciones (US FDA, 2003).

El nuevo supuesto regulador que parece seguirse ahora es: una mayor difusión de información científica, que amplíe las opciones de los consumidores, es mejor socialmente, aunque con ello se posibilite la entrada en el mercado de algún falso positivo (al menos, desde el punto de vista del más alto estándar). Así, entre las autorizadas como "declaraciones de salud" ("Health claims") se han pasado a diferenciar las "declaraciones de salud cualificadas" (Qualified health claims) (Tabla 1).

\begin{tabular}{|c|c|c|c|}
\hline \multirow{3}{*}{ 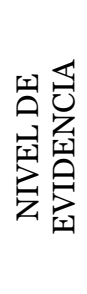 } & $\begin{array}{l}\text { Declaración de salud } \\
\quad \text { (desde 1990) }\end{array}$ & $1^{0}$ & $\begin{array}{c}\text { Probada relación causa-efecto entre } \\
\text { sustancia y salud humana } \\
\text { (Estándar: "Acuerdo científico significativo", } \\
\text { RCTs) }\end{array}$ \\
\hline & Declaraciones cualificadas de & $2^{0}$ & \multirow{2}{*}{$\begin{array}{l}\text { Evidencia creíble pero limitada } \\
\text { (Estándar: "Peso de la evidencia") }\end{array}$} \\
\hline & $\begin{array}{c}\text { salud } \\
\text { (desde 2003) }\end{array}$ & $\frac{3^{0}}{4^{0}}$ & \\
\hline
\end{tabular}

Tabla 1. Tipos de declaración de salud en los EEUU según niveles de evidencia (Elaboración propia).

\subsection{Evaluación de la compresión social de las declaraciones}

En lo que corresponde a la evaluación sobre la comprensión de las declaraciones, tanto la regulación actual europea como la norteamericana dejan claro que la redacción de la declaración se juzgará una vez haya sido suficientemente probado su

2 Véase análisis detallado de la controversia en Todt y Lujan (2017).

${ }^{3}$ Sobre otras modificaciones anteriores, e.g. Lalor y Wall (2011), y en apartado 5. 
contenido (Regulation-EU-1924/2006; ESFA-NDA, 2011; US-FDA-NLA-1990; US FDA, 2003). Si bien, mientras ambas regulaciones comparten la imposición de substanciación científica en lo que concierne al beneficio, difieren en los criterios a seguir con respecto a la cuestión sobre cómo evaluar la compresión de las declaraciones.

En el caso de Europa, para garantizar su fiabilidad y claridad, las declaraciones han de cumplir los siguientes tres requisitos (Artículo 16, Regulation-EU-1924/2006):

a) fundamentada en datos científicos;

b) ajustada a los criterios formales establecidos en el presente Reglamento;

c) comprensible y con sentido para el consumidor medio.

El primer aspecto está vinculado a la verificación de que el contenido del mensaje expresado por la declaración se corresponda con lo aceptado en ciencias de la nutrición y la salud (i.e. cuestión asociada a la evaluación de beneficios), pero también de que su redacción no fomente un consumo perjudicial o incoherente con las prácticas dietéticas generalmente recomendadas (Consideración 17) (Tabla 2).

\begin{tabular}{|c|c|c|c|}
\hline $\begin{array}{c}\text { Nutriente o sustancia } \\
\text { o categoría } \\
\text { alimentaria }\end{array}$ & $\begin{array}{c}\text { Declaración de } \\
\text { propiedades } \\
\text { saludables }\end{array}$ & Estado & $\begin{array}{c}\text { Condiciones de uso / } \\
\text { Razones para la no } \\
\text { autorización }\end{array}$ \\
\hline Vitamina B6 & $\begin{array}{c}\text { Impacto positivo en } \\
\text { el estado de ánimo, } \\
\text { especialmente en } \\
\text { mujeres }\end{array}$ & $\begin{array}{c}\text { No } \\
\text { autorizada }\end{array}$ & $\begin{array}{c}\text { No cumple con la Regulación } \\
\text { porque las condiciones } \\
\text { propuestas alentarán un } \\
\text { consumo excesivo }\end{array}$ \\
\hline
\end{tabular}

Tabla 2. Ejemplo de declaración no autorizada por la EFSA4

El segundo requisito está haciendo alusión al estándar sobre cómo el mensaje ha de ser presentado en el etiquetado (i.e. formato lingüístico o pictórico, accesibilidad de las fuentes, posición, etc.), explicitado en el mismo reglamento, aunque existen sucesivas ampliaciones y modificaciones (véase, e.g., Regulation-EU-1169/2011).

El último aspecto es el que propiamente tiene que ver con la valoración final de las autoridades evaluadoras en torno a la correcta adquisición social de lo significado por las declaraciones: solamente se autorizará el uso de declaraciones de salud "si cabe esperar que el consumidor medio comprenda los efectos benéficos tal como se expresan en la declaración” (Reglamento-EU-1924/2006).

Todos estos requisitos responden al marcado interés general europeo por garantizar el derecho de acceso del consumidor a una información alimentaria amplia y veraz (ratificado en Regulation-EU-1169/2011). Sin embargo, mientras el primer y segundo requisitos conceden a la fundamentación científica el carácter de factor principal durante la evaluación 5 , la justificación sobre la posible comprensión social de las declaraciones "no ha de constituirse según prueba estadística" (Reglamento-EU1924/2006).

4 Traducción nuestra. Fuente: EU Register of Nutrition and Health Claims. Disponible en: https://bit.ly/2Prffm1 (última consulta: 1 de febrero de 2020)

5 De hecho, se contempla la posibilidad de presentar las declaraciones con formatos distintos a los señalados en la normativa vigente, pero solo y siempre que se aporten estudios científicos que demuestren que son igualmente comprensibles a los estandarizados en ella (Regulation-EU1169/2011). 
En general, la normativa europea parte de la figura teórica de "consumidor medio". A partir de la entrada en vigor de la Directiva 84/45o/CEE del Consejo (1984), siguiendo interpretación del Tribunal de Justicia, la Comisión Europea considera tal a quien "está normalmente informado y es razonablemente atento y perspicaz, teniendo en cuenta factores sociales, culturales y lingüísticos". En nuestro caso particular, se establece que, en la determinación de la posible reacción del consumidor medio ante una declaración, serán los tribunales o autoridades competentes los que han de aplicar su propia facultad de juicio en base a la jurisprudencia (véase Consideración 15, Reglamento-EU-1924/2006).

En el caso de los Estados Unidos, el cambio visto en el enfoque epistémico de la evaluación ha conllevado un cambio en el tipo de información que ha de ser eficazmente difundida. En correspondencia con el uso de las nuevas subcategorías cualificadas de declaración, se ha implementado una nueva normativa sobre la conveniencia de su redacción. Por ejemplo, las declaraciones basadas en el segundo nivel de evidencia (véase, de nuevo, Tabla 1) deberían redactarse usando expresiones tal que "... aunque existe evidencia científica que respalda la afirmación, no es concluyente"; mientras que las correspondientes a los tercer y cuarto niveles deberían afirmarse, respectivamente, según "Alguna evidencia científica sugiere que..." e "Investigación científica muy preliminar y limitada sugiere que ...” (US FDA, 2009).

Finalmente, para evitar que la autorización de declaraciones meramente cualificadas pueda generar desconfianza en los consumidores, la regulación ha impuesto que se clarifique también qué quiere ello decir en términos de niveles de calidad de la evidencia (Figura 1).

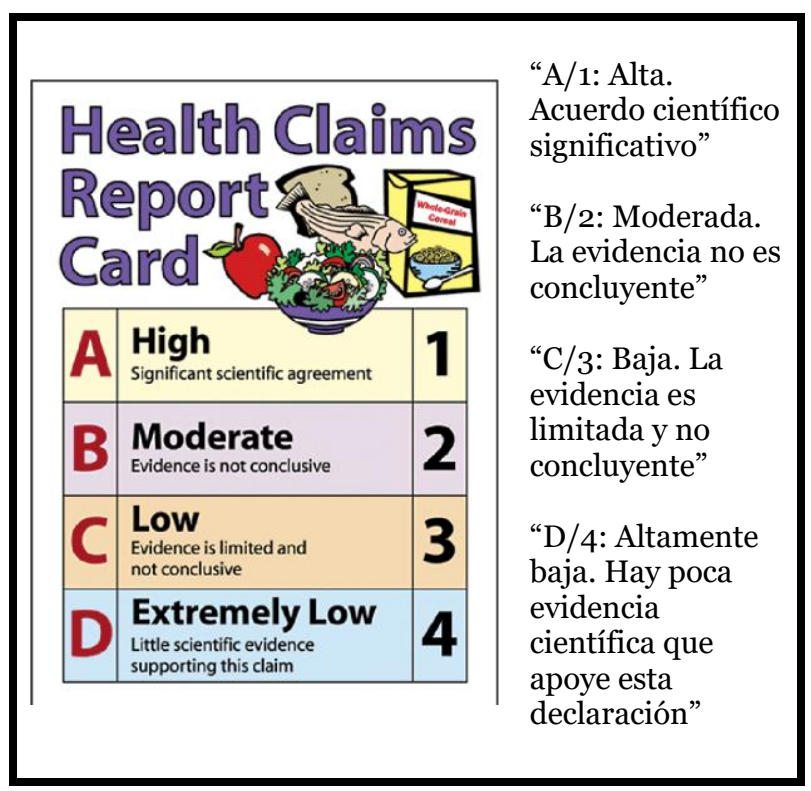

Figura 1. Tabla explicativa presente en los productos con declaraciones de salud (Traducción nuestra). Fuente: Berhaupt-Glickstein y Hallman (2017).

A diferencia de lo que ocurre en Europa, en su valoración sobre la compresión social de las declaraciones, la FDA no ha de partir de ninguna definición teórica o jurisprudencia anterior sobre el consumidor medio, sino de aquello que la ciencia pueda decir sobre la difusión social de esas nuevas consideraciones a incluir en el etiquetado. Desde la Iniciativa de 2003, se ha puesto especial énfasis en que el 
consumidor entienda cómo de fuerte es la ciencia según cada tipo de evidencia y en cómo ello puede afectar a la propia compresión del efecto sobre la salud aducido. La Iniciativa también se dirigió "a desarrollar una agenda de investigación de estudios del consumidor diseñada para identificar las formas más efectivas de presentar mejor información con base científica, veraz y no engañosa a los consumidores e identificar los tipos de información que se sabe que son engañosos para los consumidores" (FDA, 2003). ${ }^{6}$ Dicha agenda establece el tipo de cuestiones que habría de investigar el propio gobierno para asegurar el éxito de la nueva normativa. Pero, a modo informativo, también constituye un manual de sugerencias sobre los diseños metodológicos y las muestras experimentales de los estudios que podrán justificar la adecuación, al consumidor, de los distintos mensajes.

\section{La comunicación social de la ciencia en contextos reguladores}

"La evidencia científica sugiere, pero no prueba, que los cereales integrales (tres porciones o 48 gr. por día), como parte de una dieta baja en grasas saturadas y colesterol, pueden reducir el riesgo de diabetes tipo 2" es un mensaje informativo de contenido nutricional y sobre la salud. Es más, esta declaración de propiedades saludables incluye, también, información sobre la calidad de los estudios que arrojaron tal conocimiento científico. Estas declaraciones, tal y como hemos visto, se han de incluir en el etiquetado de alimentos de uso general (si así lo desean los operadores de alimentos) bajo ciertas directrices de forma y contenido impuestas por los reguladores, para que ello contribuya al logro de ciertos objetivos sociales.

Por lo tanto, una declaración de salud no solo es un acto comunicativo, en tanto que supone una transmisión intencional de información, por ejemplo, de una empresa hacia los consumidores para que compren sus productos. Es un mensaje sobre beneficios nutricionales, por lo que su contenido semántico le confiere el carácter de comunicación de la ciencia (o conocimiento experto) dirigida a un público general. Pero, aunque la ciencia sea su fuente primaria y su trasmisor una empresa (el mensaje está presente en sus productos), son las instituciones públicas las entidades garantes de su legitimidad, así como de su estandarización y adecuación a la comprensión de la audiencia objetivo. Es decir, para ayudar al consumidor en la comprensión del efecto que las elecciones alimentarias tienen en su salud, así como para protegerlo de información engañosa al respecto, los reguladores (apoyados en sus expertos) no solo son fuentes oficiales, sino que asumen la responsabilidad de su emisión. Nuestro objeto de estudio lo constituye, por tanto, este ejercicio de comunicación institucional de conocimiento sobre beneficios.

Existe innumerable bibliografía sobre comunicación social de la ciencia en general, por un lado, y de comunicación externa institucional, por otro, pero no hay estudios ni recomendaciones sobre comunicación institucional de la ciencia en tanto comunicación de beneficios. Probablemente, su desarrollo no haya sido necesario

6 Véase en Apéndice D: Consumer Studies Research Agenda -- Improving Consumer Understanding and Product Competition on the Health Consequences of Dietary Choices (actualizado en 2018): https://www.fda.gov/food/food-labeling-nutrition/consumer-healthinformation-better-nutrition-initiative-attachment-d-consumer-studies-research (última consulta: 23 de agosto 2020). 
más allá del establecimiento de las propias regulaciones o de los estudios y manuales sobre marketing y branding; o no tanto a cómo sí se ha requerido social y políticamente en torno al riesgo científico-tecnológico (Beck, 1986). Podemos basar nuestro análisis, sin embargo, en los paralelismos que encontramos entre este tipo de comunicación y de la desarrollada en contextos de riesgo. ${ }^{7}$

Los mensajes habituales de ambas actividades de comunicación institucional (riesgos y declaraciones de salud) resultan obviamente distintos si reparamos en sus respectivos contenidos específicos (riesgos vs. beneficios). También son muy distintos a nivel formal, empezando por la inevitable limitación del que nos ocupa en materia de extensión. Pero son la naturaleza de su contenido y su función los factores más relevantes para nuestro análisis, lo cuales son, en términos generales, coincidentes.

Siguiendo la definición de Senn (2008) de comunicación del riesgo, las declaraciones de salud también constituirían una forma de comunicado con información sensible de interés público. Más concretamente, parafraseando la definición de Covello (1991), podemos definir nuestro objeto de interés como el intercambio de información entre partes interesadas sobre la naturaleza, magnitud, significación o control de un beneficio nutricional para la salud. ${ }^{8}$ Además, tanto la comunicación del riesgo como la de beneficios tienen un carácter preventivo, no reactivo (e.g. como la hecha ante una crisis alimentaria) y coinciden en los objetivos comunicativos perseguidos (véase también apartado siguiente). Los específicos habituales de la comunicación institucional en torno a riesgos científico-tecnológicos son (López Cerezo, 2018: 52):

- mejorar el conocimiento del receptor

- establecer una relación de confianza entre emisor y receptor

- inducir un cambio actitudinal y/o comportamental en el receptor

No es extraño que los gobiernos contemplen la adquisición de conocimientos científico-tecnológicos como vehículo del cambio actitudinal y comportamental ciudadano, en especial, con respecto a la propia ciencia y sus productos. Esta idea se implantó en el origen teórico de lo que entendemos hoy por políticas científicas públicas. El conocido como "Informe Bush"9 defendió, entre otros supuestos, que la educación científica generalizada sería un buen medio transmisor de la naturaleza benéfica de la ciencia, esperando con ello, entre otros resultados, generar actitudes positivas hacia el consumo de los productos basados en ella (Bush, 1945). En la primera década de implantación de las políticas de promoción científica inspiradas en él, un estudio sobre la percepción y cultura científica de la sociedad estadounidense y británica, el “Informe Davis" (Withey y Davis, 1957), ayudó a asentar tal supuesto. Su

7 La comunicación institucional de riesgos (especialmente, científico-tecnológicos) es un campo de investigación y sobre buenas prácticas con cierta historia (véase, por ejemplo, en Hilgartner, 2011; López Cerezo, 2018).

8 El propio Codex Alimentarius (de la FAO-ONU) concretiza aún más una definición en esta línea: intercambio de información y opiniones entre los encargados de la evaluación de riesgos, sus gestores, industria, consumidores y aquellos actores interesados, una vez ha sido comprendida la explicación de los resultados de la evaluación y de los fundamentos de las decisiones sobre su gestión (tomada de Cámara Hurtado, 2009: 90).

9 El primer informe experto solicitado por un presidente norteamericano para aconsejar sobre las medidas gubernamentales necesarias (e.g., gran inversión) para que la ciencia pueda contribuir de manera óptima al logro de los objetivos nacionales. Su implementación en los EEUU influyó las políticas públicas I+D que luego seguirían el resto de países. 
conclusión sobre la relación alfabetización científica-actitud social se convirtió en supuesto interpretativo y guía de las políticas y la demoscopia contemporáneas al respecto. Nos referimos a la conocida en el ámbito PUS como tesis de déficit cognitivo: existe una correlación directa entre un mayor o menor conocimiento científico y más o menos bajos interés y confianza en la ciencia (Lewenstein, 1995).

Como modelo, el de déficit, incluía otros supuestos acerca de la propia ciencia y de la manera en la que esta se transfiere socialmente. Por un lado, aquello que corregía lo que se consideró una deficiencia de la población, la "cultura científica", quedaba reducida a sus aspectos más intelectuales (Jasanoff, 2005). Por otro lado, se asumía el "modelo lineal de difusión de información" (Bucchi, 2008), según el cual la comunicación científica es un proceso unidireccional de transmisión de un mensaje imperturbable desde los expertos (unánimes) a una audiencia concebida como pasiva $\mathrm{y}$ de manera general y uniforme.

Por lo tanto, respecto a la apropiación social de la ciencia, la audiencia es determinada automática y principalmente según dos factores: el mensaje (contenido intelectual) y el canal de transmisión (su forma). Es decir, la transferencia exitosa de conocimiento reside en el objeto de la transmisión, en el qué y el cómo de la información, en concreto, siempre que sea veraz y suficientemente compresible.

La importancia que con el tiempo se ha ido dando al estudio e implementación de la comunicación de riesgos siguió al comienzo y también hegemónicamente estas mismas ideas y supuestos. Si bien, tal enfoque se ha ido adaptando e, incluso, sustituyendo. En parte, su evolución ha sido resultado del reconocimiento de los especialistas y las instituciones de que la concepción clásica sobre comunicación en general seguida era simplificadora e ingenua (López Cerezo, 2018).

El modelo clásico de comunicación de la ciencia ofrece una visión que desatiende la compleja heterogeneidad de los procesos involucrados en ella, pero también del público y los emisores (tanto colectiva como individualmente), así como de sus respectivos contextos. Por un lado, la adquisición social de conocimiento, la delegación de confianza de la ciudadanía, su actitud ante la ciencia y la consecuente toma de decisiones se desarrollan a través de procesos complejos en los que influyen diversas configuraciones de experiencias personales, sistemas de creencias y de valores (sociológicos, culturales, etc.) -incluidos los vehiculados por el progreso tecnocientífico (cuyos ejemplos a menudo han sido visiblemente negativos)-, y no solo los recursos intelectuales (Lewenstein, 1995; Atienza y Luján 1997). De ahí que, e.g., la audiencia se pueda considerar siempre activa, al menos, en su interpretación del mensaje, del emisor y hasta del canal (Castells, 1997). Ello se ha corroborado con las encuestas sobre percepción social de la ciencia desde finales del s.XX (véase Durant et al. 2000; Godin y Gingras, 2000; Bauer et al., 2007).

Por otro lado, como los estudios sobre controversias científicas (STS) han mostrado, la ciencia no habla con una sola voz, sino que la tradicionalmente supuesta opinión legítima en cuestiones científico-tecnológicas, a menudo, está muy dividida (Lewenstein, 1995). Asimismo, y en particular, los de comunicación científica muestran que la transferencia de conocimiento es un proceso más bien multidireccional en todos sus contextos (especializados y sociales) (Bucchi, 2008). Es más, la ciencia está constituida por información, pero también por diversos (a veces enfrentados) supuestos, valores y prácticas que confieren un carácter altamente 
complejo a todos sus productos, incluido su contenido intelectual (Jasanoff, 2005). Por lo tanto, ni el modelo de déficit cognitivo ni el lineal de difusión se han correspondido ni se corresponden necesariamente o siempre con la realidad de las relaciones entre ciencia y sociedad.

En el caso específico de la comunicación de riesgos, han surgido alternativas teóricas al enfoque clásico motivadas por estos hallazgos. Según las más destacadas, el proceso de transmisión interna y externa de información sobre riesgos es multinivel y altamente interactivo. Sus fuentes, emisores, transmisores y receptores (ya sean expertos, reguladores, medios, público, etc.) pueden variar según estén diseminados como actores posibles a lo largo de los procesos de evaluación, gestión y comunicación social. Asimismo, se reconoce la heterogeneidad de los mensajes, los cuales se ven sucesivamente codificados y decodificados, distorsionados o modificados, intencionalmente o no, por los distintos actores y en virtud de sus creencias, actitudes, intereses, etc. (véase Renn, 2008)

Desde un punto de vista práctico, algunos especialistas defienden que el reconocimiento de la complejidad socio-técnica en la que se sitúa la comunicación institucional del riesgo contribuye a generar estrategias comunicativas más exitosas. Según Hilgartner (2011), por ejemplo, al menos, ello invita a que estas estén basadas en análisis más contextualizados sobre los factores y agentes implicados y a que se involucre a actores tradicionalmente ajenos, pero que pueden ser relevantes para evaluar, gestionar y comunicar los riesgos. Es por estas últimas características que, dada la alta variedad de propuestas sobre comunicación social de riesgos en esta línea, podemos generalizar sobre ellas que siguen un modelo comunicativo más bien constructivo (Tabla 3).

\begin{tabular}{|l|c|c|}
\cline { 2 - 3 } \multicolumn{1}{c|}{} & Modelo Clásico & Modelo Constructivo \\
\hline Proceso comunicativo & Lineal & Interactivo \\
\hline Expertos (emisor) & Unánime & Variado/controvertido \\
\hline Sociedad (audiencia) & Homogénea y pasiva & Contextual-dependiente \\
\hline $\begin{array}{l}\text { Resultado (sobre impacto } \\
\text { actitudinal y } \\
\text { comportamental) }\end{array}$ & Tesis Déficit \\
\hline $\begin{array}{l}\text { Dónde ha de ponerse el } \\
\text { énfasis que dirige la } \\
\text { práctica comunicativa }\end{array}$ & $\begin{array}{c}\text { Contenido } \\
\text { intelectual y } \\
\text { compresibilidad }\end{array}$ & $\begin{array}{c}\text { Contenido intelectual y } \\
\text { contextual, compresibilidad y } \\
\text { participación de la audiencia } \\
\text { objeto }\end{array}$ \\
\hline
\end{tabular}

Tabla 3. Enfoques en la comunicación institucional de conocimiento experto. Supuestos diferenciadores (Elaboración propia).

\section{Enfoques comunicativos sobre las declaraciones de salud y su alcance}

En el apartado tercero indagamos en los detalles teóricos y procedimentales de la regulación de las declaraciones sobre propiedades saludables de los alimentos ("declaraciones de salud") de los EEUU y de la Unión Europea. Al hacerlo, 
alcanzamos nuestro primer objetivo, mostrando que hay una correlación entre los objetivos y supuestos reguladores y los procedimentales acerca de las evaluación y autorización del uso de declaraciones. De hecho, y aunque ambas regulaciones nacieron siguiendo los mismos objetivos, hemos visto que el cambio en algunos supuestos reguladores subyacentes a estos ha motivado una modificación en los criterios epistémicos del procedimiento evaluador, así como en la propia concepción de las declaraciones, en el caso norteamericano.

Por otro lado, también hemos justificado por qué, además de sus respectivas estrategias en materia de evaluación de beneficios y gestión de las declaraciones, tanto la regulación americana como la europea constituyen una forma de comunicación institucional de conocimiento experto (apartado 4). Una declaración es un mensaje concebido para ser socialmente distribuido como parte de una estrategia reguladora dirigida a que los consumidores estén informados sobre los beneficios potenciales de sus decisiones dietéticas.

A continuación, y en cumplimiento del último objetivo de nuestra investigación, analizaremos si nuestro objeto de estudio, i.e. la comunicación social de las declaraciones de salud defendida por las regulaciones escogidas, se acerca a uno $\mathrm{u}$ otro de los modelos comunicativos vistos en el apartado anterior (según los parámetros identificados como supuestos característicos clásicos o constructivos, véase Tabla 3) y en qué medida ello resulta apropiado a la luz del éxito conseguido en el cumplimiento de los objetivos reguladores.

\subsection{Análisis y otros resultados}

No es extraño que el etiquetado alimentario se haya desvelado como fuente de instrucción técnica para la ciudadanía (Todt y Luján 1997). La Comisión Europea y la FDA asumen explícitamente su influencia en el conocimiento sobre nutrición de los consumidores, así como su impacto potencial en la toma de decisiones dietéticas (FDA, 2003; Regulation-EU-1924/2006). Estas administraciones han propuesto su regulación porque consideran que la información transmitida no solo puede contribuir positivamente en esos sentidos, sino también porque, de ser errónea o malinterpretada, podría tener efectos sociales negativos, tanto en términos de salud como por generar desconfianza en los operadores alimentarios, las instituciones o la ciencia misma.

Ambas regulaciones buscan, específicamente, la adquisición de información fiable sobre salud y nutrición por parte de los consumidores y que tal conocimiento y confiabilidad incidan positivamente en el consumo de alimentos especialmente saludables. De esta forma, tanto la normativa norteamericana como la europea constituyen estrategias para comunicar conocimiento experto que responden, en general, a los objetivos habituales de una comunicación institucional en contextos controvertidos de regulación científico-tecnológica.

A este respecto, y según lo tratado en el apartado anterior, si consideramos su medio de expresión, la comunicación científica realizada a través del etiquetado de alimentos según ambas regulaciones responde, al menos esquemáticamente y en general, al modelo lineal de difusión. Se trata de un proceso básicamente unidireccional en el que el mensaje es información científica proveniente del juicio 
experto y dirigido a una audiencia general. El canal son las etiquetas de los alimentos de uso general, mientras que el contexto emisor lo constituyen los marcos reguladores respectivos y el del receptor el contexto vital y de mercado en el que se sitúen los posibles compradores.

Ahora bien, según la regulación europea, la información transmitida ha de estar conformada por conocimiento científico unánime e incuestionable. El emisor lo constituyen únicamente autoridades competentes gubernamentales y se considera como audiencia potencial al consumidor medio, el cual, es tomado como una entidad teórica predefinida uniformemente según una caracterización muy concreta.

En cambio, varios de estos eslabones del proceso comunicativo son tratados o implementados de manera diferente desde la estrategia reguladora norteamericana. Por un lado, la información técnica (especialmente, la metacientífica) adjunta a las declaraciones cualificadas de salud habilitan una imagen social de la ciencia y del propio proceso de evaluación experta, si no menos unánime, al menos más compleja y menos idealizada. Es más, desde la Food and Drug Administration Modernization Act (US-FDA-MA-1997), en los EEUU se reconocen otras potenciales autoridades en el proceso de autorización, además de la FDA, incluidos institutos científicos no gubernamentales (e.g. el National Institutes of Health o la National Science Foundation). Por otro, su búsqueda de delegar en el consumir la decisión final de aceptar una evidencia u otra y su mayor interés por dirigirse a un consumidor real (mostrado por su atención a una caracterización científica del mismo), sumado al hecho de hacer más partícipe al propio consumidor incluso en ciertas etapas de la evaluación, ${ }^{10}$ hacen de esta regulación un proceso más interactivo en general, además de consumidor-céntrico. Comunicativamente, se estaría concibiendo a la potencial audiencia como más heterogénea y activa, así como a la recepción del mensaje como un proceso más dependiente del contexto.

Por todas estas razones puede en principio inferirse que, mientras la regulación europea estaría aún plenamente situada en la perspectiva comunicativa clásica, la americana parecería estar apostando por un enfoque comunicativo cercano a modelos constructivos. Según las posturas críticas con el modelo clásico vistas, las particularidades estadounidenses deberían contribuir a un más pronunciado y efectivo cumplimiento de la mayor parte de los objetivos reguladores buscados, al menos, los comunicativos (véase apartado 4). Sin embargo, ni en Europa ni en los EEUU parece ser el caso.

Con respecto al objetivo de lograr una mayor apropiación de cultura nutricional saludable por parte del consumir, en general y aunque aún no hay ni muchos ni muy amplios estudios sobre comprensión del efecto beneficioso declarado, la mayor parte de los consumidores dicen entender las declaraciones en niveles de moderado a alto (Hieke y Grunert, 2018). Sin embargo, el fenómeno se complica bastante si reparamos en su comprensión objetiva. En ocasiones, los consumidores tienden a pensar que un alimento con declaración de salud es superior a otro en general,

10 En concreto, con la Iniciativa de 2003, también se creó un procedimiento consultivo, como paso anterior a la evaluación definitiva por parte de la FDA (llamado "Panel period"), mediante el cual tanto otras partes interesadas como el público en general pueden opinar sobre las distintas solicitudes presentadas y en torno a las distintas cuestiones a las que está sujeta la autorización de las declaraciones de salud. 
porque extienden la causalidad beneficiosa de una de sus sustancias a todas las demás que lo constituyen (Harris et al., 2011). También puede ocurrir que el consumidor crea que el producto etiquetado como tal es más beneficioso en general, i.e. que no solo es bueno por el efecto aducido en la etiqueta sino también en relación a otras condiciones de la salud (Bilman et al., 2012). Aunque puede pensarse que los malentendidos están causados por la creciente complejidad de la información requerida en las etiquetas (Edinger, 2016), algunos experimentos muestran que las inferencias erróneas sobre salud asociada a alimentos se incrementan cuando se han propuesto mensajes más simples, i.e. con menos tecnicismos (Hieke y Grunert, 2018).

Con respecto al etiquetado estadounidense, en concreto, se ha demostrado que a los consumidores les cuesta entender, además, tanto la diferencia entre las declaraciones cualificadas y no cualificadas, como las explicaciones sobre las distintas fuentes evidenciales aportadas (GAO, 2011; Berhaupt-Glickstein y Hallman, 2017). De hecho, las imposiciones sobre las redacción y categorización asociadas con la distinta evidencia a veces conducen a que el consumir haga deducciones que no podrían estar más alejadas de la realidad. Por ejemplo, como muestran Kapsak et al. (2008), por un lado, términos como "positivos" o "prometedores", relativos a los estudios científicos, pueden ser interpretados con estrategias de marketing poco honestas. Mientras, al contrario de lo que pudiera pensarse, adjetivos tal que "poco concluyentes" se asocian positivamente con modestia y sinceridad por parte del productor alimentario. Por otro, cuando las declaraciones cualificadas están vinculadas a los niveles evidenciarios más bajos, algunas personas perciben erróneamente que el producto en su conjunto es de baja calidad o, incluso, menos saludable.

Finalmente, y con respecto al objetivo de que las declaraciones influyan en la intención de compra, los resultados de los estudios disponibles no son concluyentes (Hieke y Grunert, 2018). Lo que parece estar claro es que ni siquiera llegan a hacerlo tanto como la información nutricional presente también en las etiquetas (véase en Nocella y Kennedy, 2012) (véase también, en comparación con otros factores, más abajo).

\subsection{Discusión}

Es razonable pensar, tal y como concluyen Hieke y Grunert (2018) de su revisión de los estudios empíricos disponibles, que las autoridades que han apostado por regular las declaraciones de salud habrían sido demasiado optimistas sobre el poder de estas a la hora de contribuir a generar una sociedad con hábitos alimentarios saludables. Según lo visto, ni el alto nivel en la comprensión subjetiva de los consumidores ni los malentendidos o percepciones erróneas derivados del etiquetado parecen encontrar correlación con una mayor o menor intención de compra.

A la luz de las consideraciones críticas de los estudios sobre comunicación y comprensión sociales de la ciencia sobre los modelos clásicos, y también según los hallazgos de las investigaciones empíricas sobre la compresión y consumo de las declaraciones, en nuestra opinión ambas regulaciones podrían estar fracasando debido a su aún reconocimiento de supuestos clásicos fundamentales. Ello, a pesar de las diferencias fundamentales señaladas que existen entre ambas regulaciones. 
Como vimos, según el modelo clásico, la clave para una apropiación social exitosa de cultura científica reside en que el mensaje sea fiable y comprensible, lo que encajaría con las estrategias de comunicación seguidas tanto en el caso norteamericano como europeo. La diferencia estriba en que la regulación europea pone el énfasis en el mensaje haciendo hincapié en la infalibilidad del conocimiento científico, mientras que la norteamericana -dado que ha añadido el objetivo de ampliar las opciones de compra según distintos niveles de evidencia- se ha visto obligada a acentuar su preocupación por la claridad del mensaje también en este nuevo sentido. Pero, en cualquier caso, las políticas reguladoras de ambas administraciones estarían aún motivadas según fiel reproducción de importantes asunciones presentes en la tesis deficitaria. En concreto, el incremento de la cultura poblacional sobre beneficios, su determinación de actitudes favorables hacia ciertos alimentos, y el subsiguiente esperado incremento en su consumo son tomados como procesos que se determinan de manera automática y excesivamente racional.

Pero, al igual que se ha demostrado con respecto a otros productos tecnocientíficos, las actitudes y cambios comportamentales relativos a los alimentos con declaraciones de salud son en alta medida algo que depende del receptor, y no, o no tanto, de lo que se supone sea el alimento o de lo que se afirme al respecto en el etiquetado. Las relevancia y experiencia personales previas del consumidor constituyen los factores más significativos de incidencia, según los estudios (Lähteenmäki, 2013; Hieke y Grunert, 2018), destacando entre todas las variables que el efecto aducido tenga un interés especial para él, por ejemplo, por padecer este la enfermedad que se dice paliar. Incluso son más y muy diversas las variables más bien contextuales que, precisamente, se desvelan también como causantes significativos del interés del consumidor y su posible decisión de compra. Otras variables personales, además de la salud, tienen también efecto en la percepción y toma de decisiones, e.g. la edad o el estatus económico, así como preferencias dietéticas o las creencias previas acerca de la funcionalidad aducida. Asimismo, y según los mismos estudios, no dejan de ser más importantes que las declaraciones de salud los factores que, incluso tradicionalmente, tienen relevancia a la hora de comprar cualquier otro producto: el precio, la novedad, el sabor, etc.

Por lo tanto, las regulaciones que nos ocupan fracasan, al menos y en principio, suponiendo que, a través del mero incremento en su acervo de conocimientos sobre esos alimentos, la población decidirá racionalmente consumirlos. De hecho, la incidencia del consumidor y el contexto en el éxito de tal proceso se desvela como algo mucho más complejo y alejado de los supuestos subyacentes a este tipo de regulación en general. Como hemos visto, incluso para el caso norteamericano, ni siquiera la disponibilidad pública de más información (derivada de la mayor flexibilidad de su proceso de evaluación y la consecuente facilidad en la autorización de declaraciones), ni la transferencia a los consumidores de la responsabilidad de decisión sobre aceptar o no como suficiente la substanciación científica aportada (lo que los hace más partícipes en el proceso), parecen haber resultado medidas más efectivas al respecto. 


\section{Conclusiones}

Las regulaciones sobre declaraciones de propiedades saludables de alimentos norteamericana y europea han desarrollado estrategias de comunicación de la ciencia asociada a afirmaciones sobre salud nutricional en gran medida distintas. Diferencias en los enfoques epistémicos seguidos por sus sistemas de evaluación experta y en sus subyacentes motivaciones políticas han dado lugar a las mismas. Aún con todo, mantienen los mismos objetivos comunicativos generales que se supone que contribuirán a alcanzar su también objetivo general común de lograr una sociedad más saludable: educar a la ciudadanía, incrementar su confianza y, consecuentemente, propiciar cambios actitudinales y comportamentales.

Sin embargo, a la luz de los estudios sobre la percepción de los consumidores, estas regulaciones parecen igualmente fracasar en su cometido. Como se deduce de nuestro trabajo, tal diagnóstico es inevitable en tanto que ambas estrategias de comunicación propuestas son herederas de concepciones clásicas sobre los procesos de comunicación y asimilación sociales de la información. Centran sus políticas en la declaración misma y su mejora, suponiendo, de manera excesivamente optimista, que la mera disponibilidad de declaraciones presentes en el etiquetado incidirá de manera automática y exclusivamente intelectual en los hábitos de consumo poblacionales. Asimismo, perpetúan ideas clásicas acerca del consumidor como receptor pasivo y homogéneo o sobre la (no) relevancia de su contexto. Supuestos estos fundamentales del modelo lineal de transferencia de conocimiento científico y, sobre todo, de la tesis del déficit cognitivo de la población en materia de ciencia.

Tal y como muestran los estudios empíricos, la percepción sobre los alimentos o las declaraciones de salud no depende tanto de la información científica o sobre la ciencia aportadas, como más bien de posibles filtros cognitivos y socio-culturales (ideologías nutricionales, poder adquisitivo, etc.) o intereses particulares sobre salud de los individuos que reciben o juzgan tal información, entre otros rasgos, incluso, en principio irrelevantes en relación al contenido de estas declaraciones.

Por lo tanto, ambas regulaciones errarían, al menos, en sus supuestos de partida, que desatienden aspectos que son cruciales tanto para el proceso de comunicación científica como para el de apropiación social del conocimiento. Como ya se ha demostrado tanto por los estudios demoscópicos como desde los sociales sobre comunicación científica, ni el aprendizaje, ni la generación de actitudes positivas, ni la toma de decisiones son fenómenos tan simples como parecen ser concebidos por este tipo de regulaciones.

\section{REFERENCIAS}

Atienza, J., y Luján, J.L. (1997). La imagen social de las nuevas tecnologías biológicas en España. Madrid: Centro de Investigaciones Sociológicas.

Bauer, M.W., Allum, N., y Miller, S. (2007). "What can we learn from 25 years of PUS survey research? Liberating and expanding the agenda", Public Understanding of Science, $\mathrm{n}^{0}$ 16, pp. 79-95. https://doi.org/10.1177/0963662506071287

Beck, U. (1986). Risk Society. Londres: Sage. 
Berhaupt-Glickstein, A., y Hallman, W.K. (2017). "Communicating scientific evidence in qualified health claims", Critical Reviews in Food Science and Nutrition, $\mathrm{n}^{\mathrm{o}}$ 57, p. 13. https://doi.org/10.1080/10408398.2015.1069730

Biesalski, H. et al. (2011). "26th Hohenheim Consensus Conference, (September 11, 2010)-Scientific substantiation of health claims: Evidence-based nutrition" en Nutrition Vol.27, $\mathrm{n}^{\circ} 10$, pp. S1-S20.

Bilman, E.M., E., Van Kleef, E., Mela, D.J., Hulshof, T., y VAN TRIJP, H.C.M. (2012). "Consumer understanding, interpretation and perceived levels of personal responsibility in relation to satiety-related claims", Appetite, $\mathrm{n}^{0}$ 59, pp. 912920. https://doi.org/10.1016/j.appet.2012.07.010

Boobis, A., Chiodini, A., Hoekstra, J., Lagiou, P., Przyrembel, H., Schlatter, J., Schütte, K., Verhagen, H., y Watzl, B. (2013). "Critical appraisal of the assessment of benefits and risks for foods-BRAFO Consensus Working Group”, Food and Chemical Toxicology, ${ }^{\circ}$ 55, pp. 659-675.

Bucchi, M. (2008). "Of deficits, deviations and dialogues: theories of public communication of science", en M. Bucchi y B. Trench, B. (eds.), Handbook of public communication of science and technology (pp. 57-76). New York: Routledge.

Bush, V. (1945). "Ciencia: la frontera sin fin. Un Informe al Presidente" (Oficina de Investigación y Desarrollo Científico, Gobierno de los EEUU), REDES. revista de estudios sociales de la ciencia, $\mathrm{n}^{0} 14,89-137$.

Cámara Hurtado, M. (2009). "La comunicación del riesgo en las crisis alimentarias", en C. Moreno (ed.), Comunicar los riesgos. Ciencia y tecnología en la sociedad de la información (pp. 85-114). Madrid: Biblioteca Nueva.

Castells, M. (1997). La era de la información: economía, sociedad y cultura. Madrid: Alianza.

Covello, V. (1991). "Risk comparisons and risk communication: issues and problems in comparing Health and Environmental risks", en R.E. Kasperson y P.J.M. Stallen (eds.), Communicating Risks to the Public: International Perspectives (pp. 79-124). Dordrecht: Kluwer.

Durant, J., Bauer, M.W., Gaskell, G., Midden, C.J.H., Liakopoulos, M., y Scholten, L.M. (2000). "Two Cultures of Public Understanding of Science and Technology in Europe", en M. Dierkes y C. Von Grote (eds.), Between Understanding and Trust. The Public, Science and Technology (pp. 131-156). Reading: Harwood Academic Publishers.

Edinger, W.H. (2016). "Promoting Educated Consumer Choices. Has EU Food Information Legislation Finally Matured?”, Journal of Consumer Policy, $\mathrm{n}^{0} 39$, 9-22. https://doi.org/10.1007/s10603-015-9307-3

EFSA Panel on Dietetic Products, Nutrition and Allergies (EFSA-NDA) (2011). "General guidance for stakeholders on the evaluation of Article 13.1, 13.5 and 14 health claims", EFSA Journal, Vo. 9, nº 4, p. 2135. 
EFSA-NDA, Turck, D., et al. (2017). "Scientific and technical guidance for the preparation and presentation of an application for authorization of a Health Claim" (2 revision), en EFSA Journal, Vol.15, n ${ }^{0} 1$, p. 4680.

European Parliament and Council (Regulation-EU-1169/2011). Reglamento (UE) No. 1169/2011 del Parlamento Europeo y del Consejo, de 25 de octubre de 2011, sobre la información alimentaria facilitada al consumidor, OJ L 304 (22.11.2011), p. 18.

European Parliament and Council (Regulation-EU-1924/2006). Regulation No. 1924/of the European Parliament and of the Council on Nutrition and Health Claims Made on Foods, OJ L 404 (30.12.2006), p. 12.

Godin, B. y Gingras, Y. (2000). "What Is Scientific and Technological Culture and How Is It Measured? A Multidimensional Model”, Public Understanding of Science, $\mathrm{n}^{\mathrm{o}}$ 9, 43-58. https://doi.org/10.1088/0963-6625/9/1/303

González-Díaz, C., Gil-González, D., y Álvarez-Dardet, C. (2018). "Scientific Evidence on Functional Food and Its Commercial Communication: A Review of Legislation in Europe and the USA", Journal of Food Science, Vol. 83, ${ }^{\circ} 11$, 2710-2717. https://doi.org/10.1111/1750-3841.14359

Government Accountability Office, US (GAO) (2011). Food Labellig. FDA Needs to Reassess Its Approach to protecting Consumers from False or Misleading Claims.

Harris, J.L., Thompson, J.M., Schwartz, M.B., y Brownell, K.D. (2011). "Nutritionrelated claims on children's cereals: what do they mean to parents and do they influence willingness to buy?”, Public Health Nutrition, $\mathrm{n}^{0}$ 1, 2207-2212. https://doi.org/10.1017/S1368980011001741

Hart, E.P., y Robottom, I. (1990). "The Science-Technology-Society Movement in Science Education: A Critique of the Reform Process", Journal of Research in Science Teaching, Vol. 27, no 6, 575-88. https://doi.org/10.1002/tea.3660270607

Hieke, S. y Grunert, K.G. (2018). "Consumers and health claims", en M.J. Sadler (ed.), Foods, Nutrients and Food Ingredients with Authorised EU Health Claims (pp. 19-32), Vol 3. Cambridge: Woodhead Publishing.

Hilgartner, S. (2009). "Las dimensiones sociales de la comunicación del riesgo" en C. Moreno (ed.) Comunicar los riesgos. Ciencia y tecnología en la sociedad de la información (pp. 159-170). Madrid: Bilioteca Nueva

Jasanoff, Sh. (2005). Designs on Nature: Science and Democracy in Europe and the United States. Princeton: Princeton University Press.

Jukola, S. (2019). "On the evidentiary standards for nutrition advice", Studies in History and Philosophy of Science, $\mathrm{n}^{\mathrm{0}} \quad 73, \quad 1-9$. https://doi.org/10.1016/j.shpsc.2018.05.007

Kapsak, W.R., Schmidt, D. Childs, N.M. Meunier, J., y White, C. (2008). "Consumer perceptions of graded, graphic and text label presentations for qualified health claims", Critical Reviews in Food Science and Nutrition, $\mathrm{n}^{0}$ 48, 248-256. https://doi.org/10.1080/10408390701286058 
Lähteenmäki, L. (2013). "Claiming health in food products", Food Quality and Preference, $\mathrm{n}^{0}$ 27, 196-201. https://doi.org/10.1016/j.foodqual.2012.03.006

Lalor, F., y Wall, P.G. (2011). "Health claims regulations. Comparison between USA, Japan and European Union”, British Food Journal, Vol. 113, nº 2 , 298-313. https://doi.org/10.1108/00070701111105358

Lewenstein, B.V. (1995). "Science and the Media", en S. Jasanoff et al. (eds.), Handbook of Science and Technology Studies (pp.346-360), London: Sage.

López Cerezo, J.A. (2018). La confianza en la sociedad del riesgo. Barcelona: Sello.

Luján, J.L. y Todt, O. (2020). "Standards of evidence and causality in regulatory science: Risk and benefit assessment", Studies in History and Philosophy of Science Part A, no 80, 82-89. https://doi.org/10.1016/j.shpsa.2019.05.005

Nocella, G., y Kennedy, O. (2012). "Food health claims - What consumers understand”, Food Policy, $\quad \mathrm{n}^{\mathrm{o}} \quad 37, \quad 571-580$. https://doi.org/10.1016/j.foodpol.2012.06.001

Renn, O. (2008). Risk Governance: Coping with uncertainty in a Complex World. London: Erthscan.

Todt, O., y Luján, J.L. (1997). "Labelling of Novel Food, and Public Debate”, Science and Public Policy, Vol. 24, ${ }^{0}$ 5, 319-326. https://doi.org/10.1093/spp/24.5.319

Todt, O., y Luján, J.L. (2017). "Health Claims and Methodological Controversy in Nutrition Science", Risk Analysis, Vol. 37, $\mathrm{n}^{\mathrm{0}}$ 5, 958-968. https://doi.org/10.1111/risa.12665

US Food and Drug Administration (US-FDA-NLA-1990). Nutrition Labelling and Education Act. Public Law 101-553, 104 Stat. 2353 codified at 21 USC 343 (1993).

US Food and Drug Administration (US-FDA-MA-1997). FDA Modernization Act. U.S. Public Law 105-115, 111 stat. 2296 codified at 21 USC. 301 (1997).

US Food and Drug Administration (FDA) (2003). Consumer health information for better nutrition initiative: Task Force Final Report.

US FDA Office of Nutrition and Food Labelling (2009). Guidance for Industry: Evidence-Based Review System for the Scientific Evaluation of Health Claims.

Withey, S.B., y Davis, R.C. (1957). News Media Study. Ann Arbor, Mich., Interuniversity Consortium for Political and Social Research, 1999. 